\title{
Efficacy of fungal and bacterial antagonists for controlling growth, FUM1 gene expression and fumonisin B1 production by Fusarium verticillioides on maize cobs of different ripening stages
}

\begin{abstract}
This study was carried out to examine the efficacy of two biocontrol agents (Clonostachys rosea 016, BCA1; Gram-negative bacterium, BCA5) for control of FUM1 gene expression and fumonisin B1 (FB1) production by F. verticillioides FV1 on maize cobs of different ripening stages: R3, Milk (0.985 aw); R4, Dough (0.976 aw); R5, Dent (0.958 aw). Initially, temporal studies on FUM1 gene expression and FB1 production were performed on maize kernels for up to 14 days. This revealed that day 10 was optimum for both parameters, and was used in the biocontrol studies. Maize cobs were inoculated with 50:50 mixtures of the pathogen:antagonist inoculum and incubated in environmental chambers to maintain the natural aw conditions for ten days at 25 and $30{ }^{\circ} \mathrm{C}$. The growth rates of $\mathrm{F}$. verticillioides FV1, the relative expression of the FUM1 gene and FB1 production were quantified. It was found that, aw $\times$ temp had significant impacts on growth, FUM1 gene expression and FB1 production by $\mathrm{F}$. verticillioides $\mathrm{FV} 1$ on maize cobs of different maturities. The fungal antagonist (BCA1) significantly reduced FB1 contamination on maize cobs by $>70 \%$ at 25 ${ }^{\circ} \mathrm{C}$, and almost $60 \%$ at $30{ }^{\circ} \mathrm{C}$ regardless of maize ripening stage. For the bacterial antagonist (BCA5) however, FB1 levels on maize cobs were significantly decreased only in some treatments. These results suggest that efficacy of antagonists to control mycotoxin production in ripening maize cobs needs to take account of the ecophysiology of the pathogen and the antagonists, as well as the physiological status of the maize during silking to ensure effective control.
\end{abstract}

Keyword: Biocontrol; Fusarium; Fumonisin B1; FUM genes; Maize cob; Maturity stages; Silking 